\title{
Risk Regulation in Brazil: A General Equilibrium Model*
}

\author{
Aloísio Pessoa Araújo** \\ José Valentim M. Vicente ${ }^{* * *}$
}

\begin{abstract}
In the last few years, regulating agencies of many countries, following recommendations of the Basel Committee on Banking Supervision, have compelled financial institutions to maintain minimum capital requirements to cover market and credit risks. The capital charge to cover market risk is a function of a metric known as Value-at-Risk (VaR). This paper investigates the consequences of such practices on prices, volatilities and bankruptcy probability by considering two features of the Brazilian framework: variable risk constraint multiplier and heterogeneous beliefs between financial institutions and regulating agencies.
\end{abstract}

Keywords: Capital Requirements, Basel Capital Accord, VaR, Banking Regulation.

JEL Codes: G12, G18, G20, C68.

${ }^{*}$ Submitted in May 2005. Revised in March 2006. The authors thank Caio Ibsen Rodrigues de Almeida for his comments. The opinions expressed herein are those of the authors and not necessarily those of the Central Bank of Brazil.

${ }^{* *}$ IMPA and FGV.

${ }^{* * *}$ IMPA and Central Bank of Brazil. E-mail: jvalent@impa.br

Brazilian Review of Econometrics v. $26, \mathrm{n}^{\circ} 1$, pp. $3-29$ May 2006 


\section{Introduction}

Banking crises have continually occurred throughout time. Usually, due to the connection of banks to different economic branches, the bankruptcy of a financial institution is more detrimental to society than that of a nonfinancial one.

In an attempt to reduce the frequency and intensity of such crises, some regulations have been proposed for this sector. ${ }^{1}$ The major regulation is certainly the Basel Agreement, which resulted from a process under the heading of the Basel Committee on Banking Supervision.

The Basel Committee was set up in 1974 under the auspices of the Bank for International Settlements (BIS) by the central banks of the G10 members. ${ }^{2}$ The main aim of the Committee is to strengthen cooperation between financial supervisors. It should be highlighted that the Committee itself does not have any superior authority over governments, and therefore, its recommendations do not have legal force.

In December 1987, the Committee submitted a document to be considered by the member countries, establishing minimum capital requirements for credit risk. In July 1988, after approval by the G10 member countries, this document (known as the Basel Capital Accord or as the 1988 Basel Accord) was released to banks. Since then, the recommendations of this agreement have been gradually introduced not only into the member countries but also into all countries whose banks are internationally active.

Basically, the 1988 Basel Accord imposes a capital requirement of at least $8 \%^{3}$ of the risk-adjusted asset, defined as the sum of asset positions multiplied by asset-specific risk weights.

The second step was to define criteria for capital requirements to account for market risk. So, in January 1996, the Amendment to the Capital Accord to Incorporate Market Risks (Basel Committee on Banking Supervision, 1996a) ${ }^{4}$ set the minimum capital requirement for a financial institution as the sum of a capital charge to cover credit risk (at least $8 \%$ of the risk-weighted asset) and another charge to cover market risk.

In order to meet the requests of the financial industry, the Basel Accord Amendment of 1996 allowed the use of internal or standard models to gauge market risk. In a standard model, the regulatory authority defines the criteria for minimum

\footnotetext{
${ }^{1}$ There is no consensus agreement in the academic literature on the reasons for banking regulation. The two major reasons are: the risk of systemic crises and the inability of depositors to monitor banks. See Santos (2000) for a review of the literature on theoretical explanations for banking regulation.

${ }^{2}$ The current members of the Basel Committee are: Belgium, Canada, France, Germany, Italy, Japan, Luxembourg, Netherlands, Spain, Sweden, Switzerland, England, and the United States.

${ }^{3}$ In Brazil, the capital requirement is $11 \%$ instead of $8 \%$.

${ }^{4}$ For an overview on the Amendment to the Capital Accord to Incorporate Market Risk, see the Basel Committee on Banking Supervision (1996b).
} 
capital requirement that should be met by financial institutions. The internal model, however, gives banks the option to use their own risk measurement models to determine their capital charge. Nevertheless, in order to use this model, banks must fulfill a series of requirements. From the quantitative point of view, the $99 \%$ confidence interval Value-at-Risk $(\mathrm{VaR})^{5}$ over a 10-day horizon is used as the basis for calculating market risk. The capital requirements to cover market risk should equal the maximum between: (i) the average VaR on the previous 60 business days multiplied by a factor (known as multiplier), and (ii) the previous day's VaR. However, as the factor is always larger than three, ${ }^{6}$ the value specified in item (i) is almost always larger than the value stipulated in item (ii).

Although Brazil is not a member of the Basel Committee, its banking system follows the principles established by the Basel Accord. In 1994, the National Monetary Council (CMN), through Resolution $2,099,{ }^{7}$ took the first step towards adapting the Brazilian financial system to the international standards outlined by the Committee. This rule established that all financial institutions have to hold a minimum total capital equal to $11 \%$ of their risk-adjusted assets.

The rules for calculating the required net worth have been changing over time in order to increase their efficiency and to include several types of risks. Up to the end of 2005, financial institutions were required to allocate capital to cover the credit risks of their assets (with a different treatment for credit risk of swap agreements), to cover currency risks and gold investment risks and operations in Reais with fixed interest rates. However, there is yet no capital requirement for covering market risks related to stocks and commodities. ${ }^{8}$

The capital charge necessary to cover credit risk follows a model that closely resembles the one proposed by the 1988 Basel Accord. On the other hand, the charge necessary to cover market risk includes two risk factors: (i) currency and gold, and (ii) fixed interest rates.

Coverage of currency risk follows the standard model. In short, capital requirement to cover currency risk corresponds to $50 \%$ of the net worth of operations involving gold and assets and liabilities denominated in foreign currency. ${ }^{9}$

For the coverage of market risk of fixed interest rates, the Central Bank of

\footnotetext{
${ }^{5} \mathrm{VaR}$ is a risk metric proposed by the J.P. Morgan Bank in 1994 and represents the maximum loss to which a portfolio is subject for a given confidence interval and time horizon. For instance, a one-day $99 \% \mathrm{VaR}$ of $\mathrm{R} \$ 10$ million means that there is only 1 in 100 chance of the portfolio loss to exceed $\mathrm{R} \$ 10$ million at the end of the next business day. Undeniably, the widespread use of VaR-based risk management models results from the fact that this risk metric is easy to interpret. For an overview of VaR, see, for instance, Duffie and Pan (1997).

${ }^{6}$ In Brazil, the multiplication factor ranges between 1 and 3.

${ }^{7}$ Banking regulation rules in Brazil can be obtained from the Central Bank of Brazil (BACEN) website.

${ }^{8}$ BACEN's Communiqué 12,746, dated December 9, 2004 sets the end of 2005 as the deadline for the regulating agency to lay down guidelines for capital requirement to cover market risk that are not provided by the current regulations.

${ }^{9}$ See BACEN's Circular 3,229 dated March 26, 2004.
} 
Brazil (BACEN) used an intermediate approach between the standard and internal models. ${ }^{10}$ The capital charge necessary to cover this type of risk is calculated according to the Committee's guidelines, i.e., the maximum value between the VaR over the previous 60 business days multiplied by a factor and the previous day's VaR. Nevertheless, the rule established by BACEN includes two different aspects: (i) the parameters for VaR calculation (covariance matrix of the assets) are stipulated by BACEN on a daily basis, and (ii) the VaR multiplier is a decreasing function of market volatility, i.e., the larger the market turbulence, the lower the multiplier. ${ }^{11}$

Regardless of legal requirements, several financial institutions have recently adopted internal VaR-based models for market risk management. Most of this self-discipline process was a demand from stockholders and investors who were concerned with the increase of volatility in a globalized world and who wanted transparency in the management of their resources. Nowadays, even in emergent countries like Brazil, all banks with some market activity calculate their VaR on a daily basis.

The aim of the present study is to assess the economic implications of VaRbased risk management by means of simulations of a general equilibrium model. Since our aim here is not to assess the consequences of adopting minimum capital requirements for credit risk, we consider that there is no allocation of capital to cover such risk. ${ }^{12}$ The model used is similar to the one proposed by Daníelsson et al. (2004). However, to analyze the effects of peculiar rules established by BACEN, we implemented two changes to the above-mentioned model, namely: (i) the covariance matrix of the assets is defined by the regulating agency, and may therefore not match the market expectation and (ii) the multiplier associated with the VaR constraint is a decreasing function of market volatility. The purpose of the variable factor is to prevent high capital requirements after economic crises (see Arcoverde (2000)).

The consequences of the first change are often ambiguous and depend on how BACEN estimates the covariance matrix. Predicting the behavior of the economy is only possible under some special circumstances. For instance, if BACEN overestimates the volatility of all risky assets, then the negative effects of VaRbased capital requirement shown in Daníelsson et al. (2004) (namely: decrease in equilibrium price and increase in volatility) are enhanced. This suggests that the internal model can be more accurate in estimating market risk than the standard model. ${ }^{13}$

\footnotetext{
${ }^{10}$ Arcoverde (2000) gives an in-depth description of the method used by BACEN for the establishment of regulations for market risk of fixed interest rates.

${ }^{11}$ See BACEN's Circular 2,972 dated March 23, 2000 as well as its Technical Note.

12 Jackson et al. (1999) review the literature on the effects of minimum capital requirements for credit risk, as established by the 1988 Basel Accord.

${ }^{13}$ BACEN's Communiqué 12,746 sets the end of 2007 as the deadline for the regulating agency to establish the eligibility criteria for the adoption of internal models for market risk and for the
} 
The advantage of the second change is that it guarantees equilibrium in critical situations, which does not occur in the original model proposed by Basel.

In opposition to the academic consensus that VaR is not an appropriate risk measure, we will see that when it is used with regulatory purposes, it reduces total bankruptcy probabilities, defined as the aggregate bankruptcy probabilities of all financial agents.

In addition to this introduction, this paper is structured as follows. The next section reviews the literature on the economic consequences of using VaR as a standard model for market risk management. Section 3 summarizes the model proposed by Daníelsson et al. (2004) showing the previously mentioned adaptations. Section 4 shows the specifications of the model dynamics. Section 5 presents the simulation results and Section 6 concludes.

\section{Review of the Literature}

After the implementation of VaR as the standard procedure for market risk management in the second half of the last decade, a wide range of academic studies (either empirical or theoretical) have been carried out in order to assess the economic consequences of such practice.

Basak and Shapiro (2001) investigated the implications of the investment decision problem when the trader is subject to an exogenous VaR limit. They showed that agents who suffer such restriction divide adverse states of nature into two classes: bad states and intermediate states. Since these agents are only concerned with the probability of loss and not with its magnitude, they opt to protect themselves against intemediate states of nature and become completely vulnerable to bad states. As a result, the expected loss, considering a loss occurred, is larger for the agents that manage risk by means of a VaR model than for those who do not manage the market risk at all. To control the magnitude of losses, the authors suggest the Expected Shortfall ${ }^{14}$ as an alternative risk metric, i.e., the expected loss, considering there was a loss. With this metric, the undesirable effects of a VaR-based risk management are eliminated.

Daníelsson and Zigrand (2003) used a two-period economy with a continuum of financial institutions characterized by a constant absolute risk aversion coefficient and subject to a VaR constraint They showed that:

- Optimal risk sharing is impaired;

- If all financial institutions are regulated (i.e., if they must satisfy the risk constraint), an equilibrium might not exist;

- Volatility of positive-beta assets in a regulated economy is greater than in an unregulated economy (i.e., an economy where there are no risk limits);

validation of these models.

${ }^{14}$ For further information about Expected Shortfall, see Acerbi and Tasche (2002). 
- Prices of positive-beta assets in a regulated economy are lower than prices of assets in an unregulated economy;

- Liquidity in a regulated economy is smaller than in an unregulated economy.

Daníelsson et al. (2004) used a numerical simulation to extend the model proposed by Daníelsson and Zigrand (2003) to a multiperiod environment, and assessed the intensity of adverse impacts of VaR-based risk constraint.

Leippold et al. (2003) considered the implications of VaR-based risk management for a continuous-time economy with intermediate consumption, stochastic opportunity set and heterogeneous attitude towards risk. By using asymptotic approximation methods, they showed that VaR-based risk management can lead banks to increase their exposure to risk in highly volatile states of nature. However, the effects on volatility and expected asset return are ambiguous, depending on the dynamics of the model. On the other hand, the interest rate will always be lower and the Sharpe ratio will always be greater in a regulated economy.

Cuoco and Liu (2004) analyzed the dynamics of the investment and VaR reporting problems faced by financial institutions that are subjected to a VaR-based risk constraint, following the internal modeling approach, considering the effects of adverse selection and moral hazard. They showed that when institutions which regularly underreport its true VaR (the accuracy of the risk measurement model is checked by backtesting) are punished, internal models can be very effective not only in curbing portfolio risk, but also in inducing truthful revelation of this risk.

\section{The Model}

Consider an infinite-period economy constructed by the sequence of two-period economies as proposed by Daníelsson et al. (2004). Time is discrete and indexed by $t \in T=\{0,1,2, \ldots\}$. At each period $t$ the agents (financial institutions) invest in $N+1$ assets with maturity at $t+1$. Asset 0 is risk-free and yields payoff $d_{0, t+1}$ at $t+1$. The risky assets are non-redundant and promise to yield a payoff at $t+1$

$$
\boldsymbol{d}_{t} \equiv\left(\begin{array}{c}
d_{1, t+1} \\
\vdots \\
d_{N, t+1}
\end{array}\right)
$$

which conditioned on the information available up to $t$ follows a Gaussian distribution.

Let $q_{i t}$ be the price of asset $i$ at $t$. The return on asset $i$ between periods $t$ and $t+1$ is defined as

$$
R_{i, t+1} \equiv \frac{d_{i, t+1}}{q_{i t}}
$$

There is a large number of small agents with a constant absolute risk aversion (CARA) equal to $h$. The population of agents is such that $h$ is uniformly dis- 
tributed on the interval $[\ell, 1]$. To guarantee that all agents are risk-averse, let us suppose that $\ell>0$.

Let $x_{t}^{h}$ and $y_{i t}^{h}$ be the number of units of the risk-free asset and of the risky asset $i$, respectively, held by financial institution $h$ between periods $t$ and $t+1$. Then the wealth of agent $h$ at time $t+1$ is

$$
W_{t+1}^{h} \equiv x_{t}^{h} d_{0, t+1}+\sum_{i} y_{i t}^{h} d_{i, t+1}
$$

Agents have a very short time horizon, so they choose the portfolio that maximizes the expected value of the wealth utility in the next period subject to budget and risk constraints.

Admit that there is a fixed, deterministic time-invariant net supply of $\theta_{i}$ units of the $i^{\text {th }}$ risky asset. Let $\theta$ be the vector that represents the aggregate endowments of the risky assets, i.e.,

$$
\theta \equiv\left(\begin{array}{c}
\theta_{1} \\
\vdots \\
\theta_{N}
\end{array}\right)
$$

For reasons that will be clearer further ahead, the net supply of the risk-free asset depends on $t$ and will be denoted by $\theta_{0 t}$.

Daníelsson and Zigrand (2003) showed that in this economy equilibrium prices depend only on aggregate endowment, no matter how this wealth is distributed between the agents. Therefore, we may suppose the new supply of risky assets at each period belongs to other individuals rather than to the financial institutions. ${ }^{15}$

The budget constraint between periods $t$ and $t+1$ has the following form

$$
q_{0 t} x_{t}^{h}+\sum_{i} q_{i t} y_{i t}^{h} \leq d_{0 t} x_{t-1}^{h}+\sum_{i} d_{i t} y_{i, t-1}^{h}
$$

For each $t$, the risk constraint is fixed as a uniform upper limit for the portfolio VaR. However, this VaR is calculated according to the regulating agency's beliefs about the risky assets's payoffs. The regulating agency considers that the payoffs of the risky assets at $t+1$ conditioned on the information available at $t$ follow a normal distribution with mean $\boldsymbol{m}_{t}$ and covariance matrix $\boldsymbol{S}_{t}$. VaR is defined as

$$
V a R_{t}^{\alpha} \equiv-\inf \left\{x \in \mathbb{R} ; \mathbb{P}_{t}^{r}\left[W_{t+1}^{h}-E_{t}^{r}\left(W_{t+1}^{h}\right) \leq x\right] \leq \alpha\right\}
$$

where $\mathbb{P}_{t}^{r}$ is the probability measure corresponding to the expectations of the regulating agency at $t, E_{t}^{r}$ represents the expected value for this distribution and $\alpha$ is the significance level adopted (the probability of losses exceeding the VaR). ${ }^{16}$

\footnotetext{
${ }^{15}$ The same assumption applies to the supply $\theta_{0 t}$ of the risk-free asset.

${ }^{16} \mathrm{VaR}$ when defined by equation 1 is known as relative VaR, while the absolute VaR is defined as $\operatorname{VaR}_{t}^{\alpha}=-\inf \left\{x \in \mathbb{R} ; \mathbb{P}_{t}^{r}\left[W_{t+1}^{h} \leq x\right] \leq \alpha\right\}$ (see Jorion (2001)).
} 
Therefore, the risk constraint is

$$
\operatorname{VaR} R_{t}^{\alpha} \leq \overline{\operatorname{VaR}}
$$

where $\overline{V a R_{t}}$ is a VaR limit set by the regulating agency that depends on a market volatility index. By using normal distribution properties, the risk constraint can be rewritten as an exogenous upper limit for the portfolio variance ${ }^{17}$

$$
\boldsymbol{y}_{t}^{h^{\prime}} \boldsymbol{S}_{t} \boldsymbol{y}_{h}^{t} \leq \bar{\nu}_{t}
$$

where parameter $\bar{\nu}_{t}$, called severity of the risk constraint, ${ }^{18}$ depends on $\alpha$ and $\overline{V a R_{t}}$, and $\boldsymbol{y}_{t}^{h^{\prime}}=\left(y_{1 t}^{h}, \ldots, y_{N t}^{h}\right)$ is the portfolio of risky assets.

The investment problem of the financial institution $h$ at date $t$ can then be written as

$$
\begin{array}{cc}
\operatorname{Max} & E_{t}^{a}\left(u^{h}\left(W_{t+1}^{h}\right)\right) \\
\left(x_{t}^{h}, \boldsymbol{y}_{t}^{h^{\prime}}\right) & q_{0 t} x_{t}^{h}+\sum_{i=1}^{N} q_{i t} y_{i t}^{h} \leq d_{0 t} x_{t-1}^{h}+\sum_{i=1}^{N} d_{i t} y_{i, t-1}^{h} \\
\text { s.a. } & \boldsymbol{y}_{t}^{h^{\prime}} \boldsymbol{S}_{t} \boldsymbol{y}_{h}^{t} \leq \bar{\nu}_{t}
\end{array}
$$

where $E_{t}^{a}$ is the expected value with respect to the agents' belief at $t .^{19}$ In an unregulated economy, risk constraint is not present, i.e., $\bar{\nu}_{t}=\infty$ for all $t$. The next lemma guarantees the existence and unicity of the solution to the problem of financial institutions.

Lemma 1 For all $h$ and all $t$, the problem of financial institution $h$ at time $t$ has only one solution.

In each period $t$, an equilibrium for the economy in question is an asset price vector $\left(q_{0 t}, q_{1 t}, \ldots, q_{N t}\right)=\left(q_{0 t}, \boldsymbol{q}_{t}{ }^{\prime}\right)$ and an application $h \in[\ell, 1] \mapsto\left(x_{t}^{h}, \boldsymbol{y}_{t}^{h^{\prime}}\right)$, such that

1. $\left(x_{t}^{h}, \boldsymbol{y}_{t}^{h}\right)$ solves the problem of the financial institution $h$ at time $t$ when asset prices are equal to $\left(q_{0 t}, \boldsymbol{q}_{t}{ }^{\prime}\right)$;

2. Market clearing, i.e., $\int_{\ell}^{1} \boldsymbol{y}_{t}^{h} \mathrm{~d} h=\theta$ and $\int_{\ell}^{1} x_{t}^{h} \mathrm{~d} h=\theta_{0 t}$.

\footnotetext{
${ }^{17}$ Indeed, denoting the accumulated standard normal distribution function by $\Phi(\cdot)$, the risk constraint boils down to a volatility constraint: $\operatorname{VaR} R_{t}^{\alpha} \leq \overline{V a R_{t}} \Leftrightarrow \Phi\left(\frac{\overline{V a R_{t}}}{\mathrm{dp}^{r}\left(W_{t+1}^{h}\right)}\right) \geq \alpha \Leftrightarrow$ $\operatorname{dp}^{r}\left(W_{t+1}^{h}\right) \leq \frac{\overline{\operatorname{VaR}_{t}}}{\Phi^{-1}(\alpha)} \Leftrightarrow \operatorname{Var}^{r}\left(W_{t+1}^{h}\right) \leq\left(\frac{\overline{V^{-1} R_{t}}}{\Phi^{-1}(\alpha)}\right)^{2} \equiv \bar{\nu}_{t}$, where $\mathrm{dp}^{r}$ and $\operatorname{Var}^{r}$ represent the standard deviation and variance with respect to regulating agency's beliefs.

${ }^{18}$ It would be more appropriate to say nonseverity parameter as the larger the $\bar{\nu}_{t}$, the less strict the regulating agency.

${ }^{19}$ The agents' beliefs are homogeneous, that is, all of them expect the same behavior from the risky assets.
} 
The investment problem of financial institutions and consequently the problem to find the equilibrium prices are not so simple. We should therefore begin with a particular case. More specifically, we will first analyze an economy in which there exists only one risky asset. In this situation, the matrix algebra consists of operations with real numbers, which substantially facilitate the calculations.

Proposition 1 Consider an economy with only one risky asset, i.e., $N=1$. Let $\left(\mu_{t}, \sigma_{t}\right)$ be the agents' beliefs about the mean and variance of the risky asset payoff between $t$ and $t+1$, and $\theta$ the net supply of this asset (fixed and deterministic). Let $s_{t}$ be the expectation of the regulating agency about the variance of the risky asset. Suppose that $R_{t+1}>r_{0, t+1}$, where $r_{0, t+1}$ is the risk-free asset return between $t$ and $t+1$ (this value is known at $t$ ) and $R_{t+1}$ is the risky asset return in the same period. The solution to the problem of the financial institution $h$ at time $t$, $\left(x_{t}^{h}, y_{t}^{h}\right)$, when the risky asset price is $q_{t}$, is given by:

$$
y_{t}^{h}= \begin{cases}\frac{1}{h} \sigma_{t}^{-1}\left(\mu_{t}-r_{0, t+1} q_{t}\right) & \text { if } h \geq \sqrt{\frac{\rho_{t}}{\bar{\nu}_{t}}} \\ \sqrt{\frac{\bar{\nu}_{t}}{s_{t}}} & \text { if } h<\sqrt{\frac{\rho_{t}}{\bar{\nu}_{t}}}\end{cases}
$$

where $\rho_{t}=\left(\mu_{t}-r_{0, t+1} q_{t}\right)^{2} \sigma_{t}^{-2} s_{t}$.

In any case, $x_{t}^{h}=\frac{1}{q_{0 t}}\left(d_{0 t} x_{t-1}^{h}+d_{t} y_{t-1}^{h}-q_{t} y_{t}^{h}\right)$, where $d_{t}$ is the risky asset payoff at $t$.

Note that the introduction of the risk constraint prevents optimal risk-sharing since all institutions with CARA less than or equal to $\sqrt{\frac{\rho_{t}}{\bar{\nu}_{t}}}$ choose the same portfolio.

After solving the problem of the financial institutions, the market clearing condition automatically provides the equilibrium prices, as presented in the following proposition:

Proposition 2 Under the same conditions of Proposition 2, the equilibrium price of the risky asset at date $t$ is

$$
q_{t}=\frac{1}{r_{0, t+1}}\left(\mu_{t}-\Psi_{t} \sigma_{t} \theta\right)
$$

where $\Psi_{t}$ is the market price of risk scalar at date $t$ (Danielsson and Zigrand, 2003).

Denoting the non-principal branch of the Lambert correspondence ${ }^{20}$ by $F(\cdot)$, one has that

$$
\Psi_{t}= \begin{cases}\frac{1}{\ln \ell^{-1}} & \text { if } 0 \leq \kappa_{t}+\ell \\ -\frac{\kappa_{t} F\left(-\left(\kappa_{t}+\ell\right) e^{-1}\right)}{\kappa^{2}} & \text { if } \ell \ln \ell^{-1}<\kappa_{t} \leq 1-\ell\end{cases}
$$

\footnotetext{
${ }^{20}$ The non-principal branch of the Lambert's correspondence is the inverse of the function $f:(-\infty,-1] \mapsto\left[-e^{-1}, 0\right)$ defined by $f(x)=x e^{x}$
} 
where $\kappa_{t}=\theta \sqrt{\frac{s_{t}}{\bar{\nu}_{t}}}$. An equilibrium fails to exist if $\kappa_{t}>1-\ell$.

After analyzing this particular case, we will now turn our attention to a more general situation in which there are $N$ risky assets. The propositions in the sequel characterize the solutions to the problems of the financial institutions and of finding equilibrium prices.

Proposition 3 Let $\left(x_{t}^{h}, \boldsymbol{y}_{t}^{h}\right)$ be the solution to the problem of financial institution $h$ at time $t$ when the price vector of risky assets is equal to $\boldsymbol{q}_{t}$. Let $\left(\mu_{t}, \boldsymbol{\Sigma}_{t}\right)$ be the agents' beliefs about the mean and covariance matrix of risky assets payoffs between $t$ and $t+1$. We have:

1. If $h \geq \sqrt{\frac{\rho_{t}}{\bar{\nu}_{t}}}$ then

$$
\boldsymbol{y}_{t}^{h}=\frac{1}{h} \boldsymbol{\Sigma}_{t}^{-1}\left(\mu_{t}-r_{0, t+1} \boldsymbol{q}_{t}\right)
$$

where $\rho_{t}=\left(\mu_{t}-r_{0, t+1} \boldsymbol{q}_{t}\right)^{\prime} \boldsymbol{\Sigma}_{t}^{-1} \boldsymbol{S}_{t} \boldsymbol{\Sigma}_{t}^{-1}\left(\mu_{t}-r_{0, t+1} \boldsymbol{q}_{t}\right)$

2. If $h<\sqrt{\frac{\rho_{t}}{\bar{\nu}_{t}}}$ then

$$
\boldsymbol{y}_{t}^{h}=\sqrt{\frac{\bar{\nu}_{t}}{\rho_{t}}} \boldsymbol{\Sigma}_{t}^{-1}\left(\mu_{t}-r_{0, t+1} \boldsymbol{q}_{t}\right)
$$

In any case $x_{t}^{h}=\frac{1}{q_{0 t}}\left(d_{0 t} x_{t-1}^{h}+\sum_{i} d_{i t} y_{i, t-1}^{h}-\sum_{i} q_{i t} y_{i t}^{h}\right)$.

Proposition 4 Suppose that $R_{i, t+1}>r_{0, t+1}$ for all $i$. Then, for the economy specified in this section, the equilibrium price of risky assets at date $t$ is

$$
\boldsymbol{q}_{t}=\frac{1}{r_{0, t+1}}\left(\mu_{t}-\Psi_{t} \boldsymbol{\Sigma}_{t} \theta\right)
$$

where $\Psi_{t}$ is given by equation 5 with

$$
\kappa_{t}=\sqrt{\frac{\theta^{\prime} \boldsymbol{S}_{t} \theta}{\bar{\nu}_{t}}}
$$

Once again, an equilibrium fails to exist if $\kappa_{t}>1-\ell$.

Intuitively, Proposition 4 shows that the regulating agency acts by changing the average effective risk aversion across all agents $(\Psi)$. Observe that the variable severity of the risk constraint guarantees the equilibrium in more general situations than in the case where $\bar{\nu}_{t}=\bar{\nu}$. For instance, in moments of crisis, $\theta^{\prime} \boldsymbol{S}_{t} \theta$ tends to increase, however, since $\bar{\nu}_{t}$ is an increasing function of the market volatility, $\kappa_{t}$ is kept under control.

Proposition 4 is a generalization of the result obtained by Daníelsson and Zigrand (2003), who considered an economy with risk constraint in which the beliefs of the agents and of the regulating agency are the same, that is, $\boldsymbol{\Sigma}_{t}=\boldsymbol{S}_{t}$ and 
$\mu_{t}=\boldsymbol{m}_{t}$ for all $t$. Comparing this economy to the one presented herein, we can infer that the first one represents the regulation via internal models and that the second one is an intermediate approach between the standard model and the internal model adopted in Brazil. The following proposition compares the equilibrium price in these two situations.

Proposition 5 Consider again an economy with only one risky asset. Suppose also that $\theta>0$. Then, if $s_{t}>\sigma_{t}$ and at least one financial institution hits the risk constraint, the risky asset price is higher when internal models, instead of the intermediate approach, are used.

Investigating a bit further, it is interesting to analyze an issue that is often neglected by the literature: Does VaR-based capital requirement reduce or not the bankruptcy probability of financial institutions? To answer this question, it is necessary to define a metric for the total bankruptcy probability, i.e., a financial fragility metric. A financial institution goes bankrupt if its wealth is negative at a given time $t$. Therefore, the probability conditioned on the information available at $t, p b_{t}^{h}$, of financial institution $h$ going bankrupt at time $t+1$ is

$$
p b_{t}^{h}=\mathbb{P}_{t}^{d}\left[W_{t+1}^{h} \leq 0\right]
$$

where $\mathbb{P}_{t}^{d}$ is the probability measure conditioned on the information available at $t$ corresponding to the distribution of payoffs of risky assets.

Thus, the metric for the total bankruptcy probability is naturally defined as

$$
p g b_{t}=\int_{\ell}^{1} p b_{t}^{h} \mathrm{~d} h
$$

To calculate $p b_{t}^{h}$ it is necessary to know $x_{t}^{h}$. According to $3 x_{t}^{h}$ depends on $q_{0 t}$, which, in equilibrium, can be obtained by the Walras' Law:

$$
q_{0 t}=d_{0 t}+\frac{\left(\boldsymbol{d}_{t}-\boldsymbol{q}_{t}\right)^{\prime} \theta}{\theta_{0 t}}
$$

Analytically, it is possible to set $\theta_{0 t}$ constant over time, as done to the net supply of risky assets. However, a computationally simpler procedure is to:

1. Keep the price of the risk-free asset constant and equal to 1 in the unregulated economy;

2. Calculate $\theta_{0 t}$ using equation 9 ;

3. Run the same simulation for the regulated economy with the $\theta_{0 t}$ obtained in the previous item.

Note that this procedure is equivalent to the existence of a monetary authority that controls the supply of risk-free assets so as to keep the interest rate constant for the unregulated economy. 


\section{Simulation Dynamics}

The key issue to the simulation of the economy specified in the previous section lies in the modeling of three major processes:

1. The data generating process (DGP) for asset payoffs;

2. The belief revision process of the agents;

3. The belief revision process of the regulator.

In choosing the DGP, our primary objective was to mirror important stylized facts regarding financial returns, in particular volatility clustering, unconditional non-normality, and the relative size difference between returns and volatility for equities. Bearing this in mind, we considered a multivariate $\operatorname{GARCH}(1,1)$ process to be a DGP. Since multivariate GARCH models are difficult to use due to the large number of parameters and their nonlinear relationships, simplifications have been proposed. Here we use an approach known as BEKK GARCH (see Santos (2002) for further details on multivariate GARCH models). Data generating was done in MATLAB ${ }^{\mathrm{TM}}$ using the function full_bekk_simulate.m of the toolbox UCSD GARCH (Sheppard (1999)).

Financial institutions do not know the DGP, then they can only infer it from historical data. Let us admit that the agents update their beliefs about the asset returns according to the Exponentially Weighted Moving Average (EWMA) method, as recommended by RiskMetrics ${ }^{\mathrm{TM}}$. Therefore, financial institutions believe that assets returns at $t+1$ conditioned on the information available at $t$ are normally distributed with mean

$$
\mu_{t}=\left(\begin{array}{c}
\mu_{1 t} \\
\vdots \\
\mu_{N t}
\end{array}\right)
$$

and covariance matrix

$$
\boldsymbol{\Sigma}_{t}=\left(\begin{array}{ccc}
\sigma_{t}^{11} & \cdots & \sigma_{t}^{1 N} \\
\vdots & \ddots & \vdots \\
\sigma_{t}^{1 N} & \cdots & \sigma_{t}^{N N}
\end{array}\right)
$$

where $\mu_{i t}$ is the expected value of $R_{i, t+1}$ and $\sigma_{t}^{i j}$ is the covariance between $R_{i, t+1}$ and $R_{j, t+1}$.

The updating rule for agents' beliefs can be expressed as follows:

$$
\left(\left(\mu_{t}, \boldsymbol{\Sigma}_{t}\right), \mathbf{R}_{t+1}\right) \mapsto\left(\mu_{t+1}, \boldsymbol{\Sigma}_{t+1}\right)
$$

where

$$
\sigma_{t+1}^{i j}=\rho \sigma_{t}^{i j}+(1-\rho)\left(R_{i, t+1}-\mu_{i t}\right)\left(R_{j, t+1}-\mu_{j t}\right)
$$


and

$$
\mu_{t+1}=\rho \mu_{t}+(1-\rho) \mathbf{R}_{t+1}
$$

The decay factor $\rho$ is set at 0.97 as recommended by RiskMetrics ${ }^{\mathrm{TM}}$.

The regulating agency revises its expectations every two months (44 business days). This window is consistent with the average periodicity of the parameters updates stipulated by BACEN's Circular 2,972. ${ }^{21}$ The regulating agency's belief is still conditional normal, however the covariance matrix and the mean payoff are estimated, respectively, by the sample covariance matrix and by the sample mean of the payoffs of the last 43 observations.

In addition to the updating of beliefs, it is necessary to establish a rule for the variable multiplier. This can be achieved by making the severity of the risk constraint, $\bar{\nu}_{t}$, an increasing function of a market turbulence index. Theoretical sophistications do not add much in this case; so, we take $\bar{\nu}_{t}$ as a linear function of the variance of a portfolio formed by the supply of risky assets, that is,

$$
\bar{\nu}_{t}=M \theta^{\prime} \boldsymbol{S}_{t} \theta
$$

where $M$ is a positive constant.

The dynamics of our model are generated in the following fashion: The economy begins with an initial arbitrary set of beliefs $\left\{\left(\mu_{0}, \boldsymbol{\Sigma}_{0}\right) ; \boldsymbol{S}_{0}\right\}$. Based on these beliefs, agents make their portfolio choices according to equations 6 and 7 . Given the portfolio choices, the aggregate demand functions can be defined. Together with the aggregate endowments, by market clearing, we can calculate the assets prices. Then, the realizations of payoffs $\left\{\boldsymbol{d}_{1}\right\}$ determines the returns $\boldsymbol{R}_{1}$ for the risky assets, and the agents and financial institutions update their beliefs. This process is repeated until the simulation ends at $t=T$.

\section{Results}

The following table shows the parameters used in the simulation. As the aim here is to show how the assets behave in a regulated economy, we consider the existence of only two risky assets. Considering $N>2$ would complicate numerical calculations without adding any important information.

\footnotetext{
${ }^{21} \mathrm{BACEN}$ revises the parameters of Circular 2,972 within no longer than one month. However, under normal market conditions, the parameters have remained unchanged, on average, for two months.
} 
Table 1

Parameters used in the simulation

\begin{tabular}{ll}
\hline Endowments & $\theta=\left(\begin{array}{l}1.9 \\
0.5\end{array}\right)$ \\
\hline Risk-free asset payoff & $r_{0, t+1}=1.00013, \forall t$ \\
\hline Lowest risk aversion & $\ell=0.0011$ \\
\hline Linear function coefficient $\bar{\nu}_{t}$ & $M=40$ \\
\hline Unconditional covariance matrix of risky assets & $\left(\begin{array}{cc}0.6 & 0.25 \\
0.25 & 0.4\end{array}\right)$ \\
\hline Unconditional mean of risky assets payoff & $\left(\begin{array}{c}1.5 \\
1.2\end{array}\right)$ \\
\hline
\end{tabular}

To make sure the results are not affected by initial conditions, the economy adjusts for 500 periods and only after that we start to record the data. Thus, date 1 corresponds to the $501 \underline{s t}$ period of the simulation.

The main numerical conclusions of the simulation are shown in a series of figures. First, we analyze the evolution of the total bankruptcy probability of an economy à la Daníelsson et al. (2004), i.e., the agents' beliefs are identical with those of the regulating agency and the risk constraint multiplier is constant or equivalently $\bar{\nu}_{t}=\bar{\nu}$ for all $t$. Then, by maintaining the agents' expectations identical with those of the regulating agency, we examine the characteristics of a variable risk constraint multiplier according to the rule defined in equation 10 . Finally, we assess the consequences of different beliefs between the agents and the regulating agency. So as not to bias the results with other information, we set, once again, $\bar{\nu}_{t}=\bar{\nu}$ for all $t$ in the last simulation.

\subsection{Regulated versus unregulated economy}

Initially, let us analyze the behavior of the total bankruptcy probability in a regulated economy and in an unregulated economy in which the agents and the regulating agency share the same beliefs and where $\bar{\nu}_{t}=\bar{\nu}=100$. The sampling period corresponds to 250 business days (approximately one year). In order to prevent simulation uncertainties from influencing the results, 1000 independent simulations were made. Thus, the reported data correspond to the mean of these simulations.

Figure A.1 shows the evolution of the mean of the asset 1 equilibrium price throughout the sampled period. The asset price in an unregulated economy is approximately $12 \%$ higher than the price in a regulated economy. Figure A.2 shows agents' forecast variance for the return of risky asset 1. Clearly, the imposition of a VaR-based risk constraint increases the expected forecast variance. These results mirror those obtained by Daníelsson et al. (2004). Intuitively, the reduction in prices and the increase in volatility occur because risk constraint causes a transfer of risky assets from the least risk-averse agents to the most risk-averse ones. But this only happens if the asset price is reduced, which implies increase in volatility, since payoffs are generated exogenously. Figure A.3 shows the estimates for the 
correlation coefficient between the risky assets in both situations analyzed. Risk regulation causes a small decrease in the correlation between assets. This suggests that VaR-based capital requirements can reduce the probability of systemic crises.

Figure A.4 plots the total bankruptcy probability. The graph shows that the total bankruptcy probability in an unregulated economy is approximately $18 \%$ higher than in a regulated one. Basically, this occurs because risk constraint forces the agents to choose portfolios that are more focused on less risky assets. This is probably one of the reasons that justify the use of the VaR with regulation instrument for risk control.

\subsection{Variable risk constraint multiplier}

Let us now analyze the economic implications when risk limit $\bar{\nu}_{t}$ depends on a market turbulence index. We ran 1000 independent simulations. The reported results correspond to the mean of these 1000 simulations. In each simulation, we first calculated prices and variances using $\bar{\nu}_{t}$ as specified by equation 10 . Afterwards, we performed a new run with the same payoffs generated in the previous run, but making the risk limit constant and equal to the mean risk limits of the 250 observations, i.e., $\bar{\nu}=\sum_{t=1}^{250} \bar{\nu}_{t} / 250$. The aim of this procedure is to compare the effects of a variable risk limit with a constant risk limit, but keeping similar capital requirement in both situations. To simplify, let us consider that the beliefs of the regulating agency and of the agents are the same.

Figure A.5 shows the equilibrium price series of asset 1. As can be observed, the variable risk limit causes a small decrease in the equilibrium price (around $0.33 \%)$. Figure A.6 shows the variance estimate in both runs. The variable risk limit introduces the undersirable effect of increasing the variance. However, this is a small shortcoming (less than 4\%). The explanation for these results is that the regulating agency must be very strict under normal market conditions when the risk limit is variable in order to maintain the same average capital requirement in both situations.

\subsection{Differences between the beliefs of the regulating agency and of the agents}

Finally, let us look at the effects on equilibrium prices and variance when the agents' beliefs do not match those of the regulating agency. To eliminate possible noises with regard to the use of a variable severity risk constraint, consider that $\bar{\nu}_{t}=100$ for all $t$. Again, the reported results correspond to the mean of 1000 simulations.

Let us first analyze two extreme cases:

$$
\text { 1. } \boldsymbol{S}_{t}=\left(\begin{array}{cc}
0.2 & 0.25 \\
0.25 & 0.1
\end{array}\right), \quad \forall t
$$




$$
\text { 2. } \boldsymbol{S}_{t}=\left(\begin{array}{cc}
0.9 & 0.25 \\
0.25 & 0.7
\end{array}\right), \quad \forall t
$$

In situation 1 , the regulating agency underestimates the volatilities, whereas the opposite is true for situation $2 .^{22}$ The simulation results in these two cases are shown in figures A.7 and A.8. In situation 1, the equilibrium prices and variances of asset 1 are respectively higher and lower than in the case in which the regulating agency and the agent share the same beliefs. The opposite occurs in situation 2 .

As a theoretical exercise, let us analyze an intermediate situation in which the updating of beliefs of the regulating agency is made according to the rule described in section 4. Figures A.9 and A.10 respectively show the equilibrium prices and the forecast variance of asset 1 . When the beliefs of agents and of the regulating agency differ, the equilibrium price and the forecast variance are higher than in a regulated economy with homogeneous expectations. This shows that the updating method used by the regulating agency underestimates the variance of asset 1 , which, as a result, causes an increase in the equilibrium price. Nevertheless, the effect on variance is detrimental. This is due mainly to imperfections in the covariance estimate between assets 1 and 2 or in variance of asset 2 .

\section{Conclusion}

The primary aim of this paper was to analyze the economic impacts of Brazilian peculiarities regarding market risk regulation of financial institutions by means of simulations of a general equilibrium model, i.e., the risk constraint multiplier depends on market volatility and heterogeneous beliefs between the regulating agency and agents. These are the major conclusions:

- When severity is variable, prices are lower and variance is higher than in the case in which severity is constant. However, these undesirable effects are negligible. On the other hand, variable severity guarantees the existence of equilibrium in periods of market turbulence. This equilibrium would not be achieved if severity were constant;

- When the regulating agency imposes its beliefs on financial institutions for calculation of the risk limit, or when the regulating agency imposes an intermediate model between the standard and internal ones in order to allocate capital to cover market risk, the effects on volatilities and equilibrium prices depend on the method used by the regulating agency to estimate the covariance matrix. This suggests that the adoption of internal models produces a more efficient equilibrium to the economy. In this regard, BACEN, by means of Communiqué 12,746, establishes deadlines for the gradual shift of

\footnotetext{
${ }^{22}$ If we generate a large number of paths for the payoff of risky assets according to the rule defined in Section 4 and using the parameters shown in Table 1, then, the variance of risky assets estimated by financial agents (via EWMA) is larger than in situation 1 and smaller than in situation 2 .
} 
Brazilian rules to an internal modeling approach. However, one should recall that this method requires much more banking supervision;

- When an intermediate model is adopted for risk regulation (which is the case of Brazil), special attention should be paid to the updating of beliefs, since, if the regulating agency overestimates the volatility of assets, there may be negative effects.

Concomitantly, we also observed that VaR-based risk regulation reduced the total bankruptcy probability, defined as the sum (integral) of bankruptcy probabilities of all financial institutions. This indicates that the regulating agency should try to balance the negative and positive effects of risk regulation. An overly strict regulation (equivalent to a low $\bar{\nu}_{t}$ ) would minimize the bankruptcy probability, but it would increase volatility and reduce equilibrium prices. On the other hand, a more flexible regulation (equivalent to a high $\bar{\nu}_{t}$ ) would have positive effects on volatility and equilibrium prices, but it would increase the bankruptcy probability of financial institutions.

\section{References}

Acerbi, C. \& Tasche, D. (2002). On the coherence of expected shortfall. Journal of Banking \& Finance, 26:1487-1503.

Arcoverde, G. L. (2000). Alocação de capital para cobertura do risco de mercado de taxa de juro de natureza prefixada. Master's thesis, Escola de Pós-Graduação em Economia, Fundação Getulio Vargas.

BACEN (2004). Brazil, Central Bank of Brazil. Contain information and rules about capital requirements in Brazil. Available at <http://www.bacen.gov.br>.

Basak, S. \& Shapiro, A. (2001). Value-at-risk based risk management: Optimal policies and asset prices. Review of Financial Studies, 14(2):371-405.

BASEL (1996a). BASEL commitee on banking supervision. Amendment to the Capital Accord to Incorporate Market Risk.

BASEL (1996b). BASEL committee on banking supervision. Overview of the amendment to the capital accord to incorporate market risk.

Cuoco, D. \& Liu, H. (2004). An analysis of VaR-based capital requirements. April. AFA San Diego Meetings.

Daníelsson, J. P., Shin, H. S., \& Zigrand, J.-P. (2004). The impact of risk regulation on price dynamics. Journal of Banking \& Finance, 28:1069-1087. 
Daníelsson, J. P. \& Zigrand, J.-P. (2003). What happens when you regulate risk? Evidence from a simple equilibrium model. Working Paper, London Scholl of Economics.

Duffie, D. \& Pan, J. (1997). An overview of value at risk. Journal of Derivatives, $4: 7-49$.

Jackson, P. C. F., Hancock, D. J., Perradin, W., Radecki, L., \& Yoneyama, M. (1999). Capital requirements and bank behaviour: The impact of the Based Capital Accord. BIS Working Paper 1.

Jorion, P. (2001). Value-at-Risk: New Benchmark for Controlling Market Risk. McGraw-Hill, New York.

Leippold, M., Trojani, F., \& Vanini, P. (2003). Equilibrium impact of value-at-risk. Working Paper, University of Zurich.

Santos, J. A. (2000). Bank capital regulation in contemporary banking theory: A review of the literature. BIS Working Paper 90.

Santos, L. C. F. (2002). Avaliação de modelos GARCH multivariados no cálculo do valor-em-risco de uma carteira de renda variável. Master's thesis, Universidade Federal do Rio de Janeiro, Instituto de Pós-Graduação em Administração.

Sheppard, K. (2004). UCSD GARCH Tollbox, version 2.0.5. Avaible at <http://www.kevinsheppard.com/research/ucsd_garch/ucsd_garch.aspx>. 


\section{Appendix A}

\section{Proof of Lemma 1}

As $\boldsymbol{S}_{t}$ is positive definite, the set $\left\{\boldsymbol{x} \in \mathbb{R}^{N}: \boldsymbol{x}^{\prime} \boldsymbol{S}_{t} \boldsymbol{x} \leq \bar{\nu}_{t}\right\}$ is compact and convex. Therefore, the constraint set of the financial institution problem is also compact and convex. Since the objective function of this problem is continuous and convex, there is only one solution.

\section{Proof of Proposition 1}

As the utility of agent $h$ is strictly increasing, the budget constraint should be bind. Therefore, the wealth of institution $h$ at date $t+1$ is given by:

$$
W_{t+1}^{h}=\left(W_{t}^{h}-q_{t} y_{t}^{h}\right) r_{0, t+1}+d_{t+1} y_{t}^{h}
$$

Since agents have a constant absolute risk aversion coefficient, without loss of generality, we can suppose that the utility of institution $h$ has the form

$$
u^{h}(x)=-\mathrm{e}^{-h x}
$$

Then, the investment problem of institution $h$ is:

$$
\begin{array}{cl}
\underset{y_{t}^{h}}{\operatorname{Max}} & E_{t}^{a}\left(-\mathrm{e}^{-h W_{t+1}^{h}}\right) \\
\text { s.a. } & s_{t}\left(y_{t}^{h}\right)^{2} \leq \bar{\nu}_{t}
\end{array}
$$

By the normality hypothesis of the payoff one has that $-h W_{t+1}^{h}$ is also normal with mean $-h\left[\left(W_{t}^{h}-q_{t} y_{t}^{h}\right) r_{0, t+1}+\mu_{t} y_{t}^{h}\right]$ and variance $h^{2}\left(y_{t}^{h}\right)^{2} \sigma_{t}$. Therefore, $-\mathrm{e}^{-h W_{t+1}^{h}}$ is log-normal with mean

$$
-\mathrm{e}^{-h\left[\left(W_{t}^{h}-q_{t} y_{t}^{h}\right) r_{0, t+1}+\mu_{t} y_{t}^{h}\right]+\frac{h^{2}\left(y_{t}^{h}\right)^{2} \sigma_{t}}{2}}
$$

Taking logarithms, eliminating the terms that do not depend on $y_{t}^{h}$ and dividing by $h$, the problem of financial institution $h$ becomes

$$
\begin{array}{cc}
\operatorname{Min}_{y_{t}^{h}} & \left(q_{t} r_{0, t+1}-\mu_{t}\right) y_{t}^{h}+\frac{h\left(y_{t}^{h}\right)^{2} \sigma_{t}}{2} \\
\text { s.a. } & s_{t}\left(y_{t}^{h}\right)^{2} \leq \bar{\nu}_{t}
\end{array}
$$

whose Lagrangean is

$$
L\left(y_{t}^{h}, \lambda_{t}^{h}\right)=\left(q_{t} r_{0, t+1}-\mu_{t}\right) y_{t}^{h}+\frac{h\left(y_{t}^{h}\right)^{2} \sigma_{t}}{2}+\lambda_{t}^{h}\left[s_{t}\left(y_{t}^{h}\right)^{2}-\bar{\nu}_{t}\right]
$$


The first-order condition is

$$
y_{t}^{h}=\left(h \sigma_{t}+2 s_{t} \lambda_{t}^{h}\right)^{-1}\left(\mu_{t}-q_{t} r_{0, t+1}\right)
$$

If $h \geq \sqrt{\frac{\rho_{t}}{\bar{\nu}_{t}}}$ then one can easily see that $y_{t}^{h}=\left(h \sigma_{t}\right)^{-1}\left(\mu_{t}-q_{t} r_{0, t+1}\right)$ satisfies the first-order condition with the risk constraint not bind $\left(\lambda_{t}^{h}=0\right)$.

If $h<\sqrt{\frac{\rho_{t}}{\overline{\nu_{t}}}}$ then the risk constraint should be active, therefore $\lambda_{t}^{h}=\frac{\mu_{t}-q_{t} r_{0, t+1}}{2 \sqrt{s_{t} \overline{\bar{\nu}_{t}}}}-$ $\frac{h \sigma_{t}}{2 s_{t}}$ implying that $y_{t}^{h}=\sqrt{\frac{\overline{\nu_{t}}}{s_{t}}}$.

By the convexity, the first-order condition is also sufficient. According to Lemma 1 the only solution to the problem of financial institution $h$ is given by equation 4 .

\section{Proof of Proposition 2}

Let $\Psi=\frac{\left(\mu-q r_{0}\right)}{\theta \sigma}$ and define the following function:

$$
f\left(\Psi_{t}\right)= \begin{cases}\Psi_{t} \ln \ell^{-} 1 & \text { if } 0<\Psi_{t}<\frac{\ell}{\kappa_{t}} \\ \frac{\kappa_{t} \Psi_{t}-\ell}{\kappa_{t}}-\Psi_{t} \ln \kappa_{t} \Psi_{t} & \text { if } \frac{\ell}{\kappa_{t}} \leq \Psi_{t}<\frac{1}{\kappa_{t}} \\ \frac{1-\ell}{\kappa_{t}} & \text { if } \Psi_{t} \geq \frac{1}{\kappa_{t}}\end{cases}
$$

The market clearing condition is equivalent to finding $\Psi$ such as $f(\Psi)=1$, that is, the equilibrium prices are given by $q=\frac{\mu-\Psi \sigma \theta}{r_{0}}$, where $\Psi$ is the solution of $f(\Psi)=1$. Solving this equation, we have:

1. If $0 \leq \kappa_{t}<\ell \ln \ell^{-1}$ then $\Psi_{t}=\frac{1}{\ln \ell^{-1}}$;

2. If $\ell \ln \ell^{-1} \leq \kappa_{t}<1-\ell$ then

$$
f\left(\frac{\ell}{\kappa_{t}}\right) \leq 1<f\left(\frac{1}{\kappa_{t}}\right)
$$

i.e., the solution is in the central branch of $f$. Therefore, we have to solve the following equation:

$$
\frac{\kappa_{t} \Psi_{t}-\ell}{\kappa_{t}}-\Psi_{t} \ln \kappa_{t} \Psi_{t}=1
$$

which is equivalent to

$$
z \ln z=-\left(\kappa_{t}+\ell\right)
$$

where $z=\kappa_{t} \Psi_{t} \mathrm{e}^{-1}$.

Equation 11 can be written as $F\left(-\left(\kappa_{t}+\ell\right) \mathrm{e}^{-1}\right)=z \ln z$. Substituting this equality in 11 one has

$$
\Psi_{t}=-\frac{\kappa_{t}+\ell}{\kappa_{t} F\left(-\left(\kappa_{t}+\ell\right) \mathrm{e}^{-1}\right)}
$$


3. If $\kappa_{t}=1-\ell$ then $\Psi_{t}$ is any real number greater than $1 / \kappa_{t}$;

4. If $\kappa_{t}>1-\ell$ then there is no $\Psi_{t}$ that satisfies the market clearing condition, i.e., an equilibrium fails to exist.

\section{Proof of Proposition 3}

Following the same procedure used for Proposition 1 one can easily see that the Lagrangean of the problem of financial institution $h$ is

$$
L\left(\boldsymbol{y}_{t}^{h}, \lambda_{t}^{h}\right)=\left(r_{0, t+1} \boldsymbol{q}_{t}-\mu_{t}\right) \boldsymbol{y}_{t}^{h}+\frac{h \boldsymbol{y}_{t}^{h^{\prime}} \boldsymbol{\Sigma}_{t} \boldsymbol{y}_{t}^{h}}{2}+\lambda_{t}^{h}\left(\boldsymbol{y}_{t}^{h^{\prime}} \boldsymbol{S}_{t} \boldsymbol{y}_{t}^{h}-\bar{\nu}_{t}\right)
$$

The first-order condition is

$$
\boldsymbol{y}_{t}^{h}=\left(h \boldsymbol{\Sigma}_{t}+2 \lambda_{t}^{h} \boldsymbol{S}_{t}\right)^{-1}\left(\mu_{t}-r_{0, t+1} \boldsymbol{q}_{t}\right)
$$

If $h \geq \sqrt{\frac{\rho}{\overline{\nu_{t}}}}$ then $\boldsymbol{y}_{t}^{h}=\frac{1}{h} \boldsymbol{\Sigma}_{t}^{-1}\left(\mu_{t}-r_{0, t+1} \boldsymbol{q}_{t}\right)$ is the solution to the problem of agent $h$ with the risk constraint not bind $\left(\lambda_{t}^{h}=0\right)$.

If $h<\sqrt{\frac{\rho}{\bar{\nu}_{t}}}$ then the risk constraint should be active, i.e, $\boldsymbol{y}_{t}^{h^{\prime}} \boldsymbol{S}_{t} \boldsymbol{y}_{t}^{h}=\bar{\nu}_{t}$. To solve the optimization problem in this case, we can substitute equation 12 in the risk constraint, find $\lambda_{t}^{h}$ and then find the optimal portfolio. This process involves serious technical difficulties since we must determine the roots of a polynomial of order $2 N$. A simpler method is to use the results obtained for the one-dimensional case and infer a possible optimizing solution $y_{t}^{h}$. Then, one should check whether this candidate satisfies the first-order condition.

When $N=1$ we show that all intitutions with active risk constraint behaved as if their CARA were equal to $\sqrt{\frac{\rho}{\bar{\nu}_{t}}}$, i.e., optimal risk sharing was impaired. Based on this observation, a natural choice for $y_{t}^{h}$ is given by equation 7 . One can easily see that this choice of $y_{t}^{h}$ satisfies the first-order condition with active risk constraint.

Again, by the convexity, the first-order condition is also sufficient. According to Lemma 1 the only solution to the problem of financial institution $h$ is given by equations 6 and 7 .

\section{Proof of Proposition 4}

We want to show that $\boldsymbol{p}_{t} \equiv \boldsymbol{\Sigma}_{t}^{-1}\left(\mu_{t}-r_{0, t+1} \boldsymbol{q}_{t}\right)=\Psi_{t} \theta$ with $\Psi_{t}$ defined by equation 5. When $0 \leq \kappa_{t} \leq \ell \ln \ell^{-1}$ (which corresponds to the case in which no institutions reach the risk constraint) or when $\kappa_{t} \geq 1-\ell$ (which corresponds to the case in which risk constraints of all institutions are active, with no equilibrium if strict inequality holds), the demonstration is trivial. Let us focus now on the intermediate situation, i.e, $\ell \ln \ell^{-1}<\kappa_{t}<1-\ell$, in other words, some institutions have an active risk constraint and others don't. 
The market clearing condition gives

$$
\int_{\ell}^{\sqrt{\frac{\rho_{t}}{\bar{\nu}_{t}}}} \sqrt{\frac{\overline{\nu_{t}}}{\rho_{t}}} \boldsymbol{p}_{t} \mathrm{~d} h+\int_{\sqrt{\frac{\rho_{t}}{\bar{\nu}_{t}}}}^{1} \frac{\boldsymbol{p}_{t}}{h} \mathrm{~d} h=\theta
$$

or

$$
\left[1-\ell \sqrt{\frac{\overline{\nu_{t}}}{\rho_{t}}}-\ln \left(\sqrt{\frac{\rho_{t}}{\overline{\nu_{t}}}}\right)\right] \boldsymbol{p}_{t}=\theta
$$

Therefore, vectors $\boldsymbol{p}_{t}$ and $\theta$ are collinear, let us make $\boldsymbol{p}_{t}=\Psi_{t} \theta$. Then $\Psi_{t}$ should satisfy the following equation:

$$
\frac{\kappa_{t} \Psi_{t}-\ell}{\kappa_{t}}-\Psi_{t} \ln \kappa_{t} \Psi_{t}=1
$$

which is identical to the equation for $\Psi_{t}$ obtained in the one-dimensional (see proof of Proposition 4 which concludes the demonstration).

\section{Proof of Proposition 5}

The demonstration is an immediate consequence of the fact that $\Psi_{t}$ (defined by equation 5 ) is a strictly increasing function of $\kappa_{t}$ on the interval $[0,1-\ell]$. 


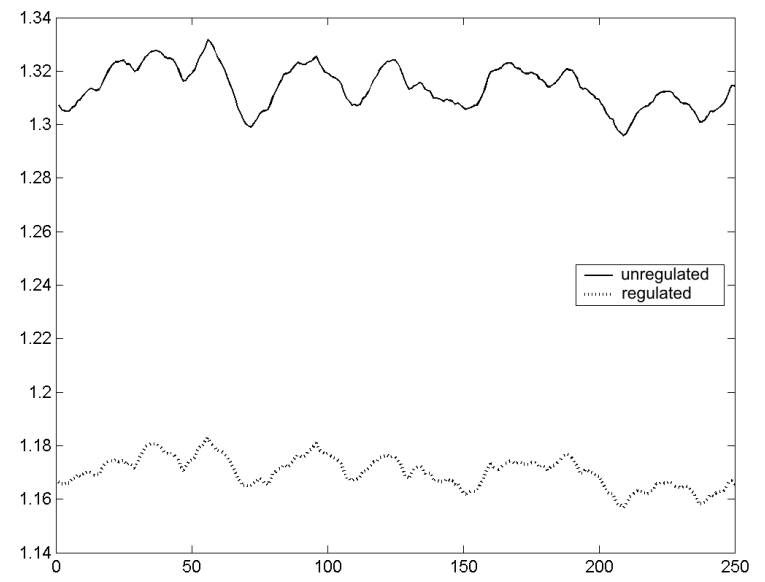

Figure A.1

Average price of asset 1 in 1000 simulations

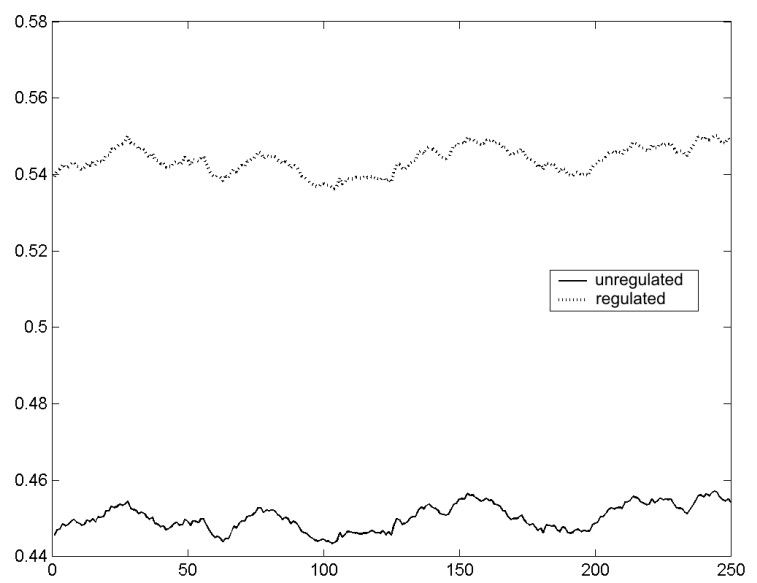

Figure A.2

Average variance of asset 1 in 1000 simulations 


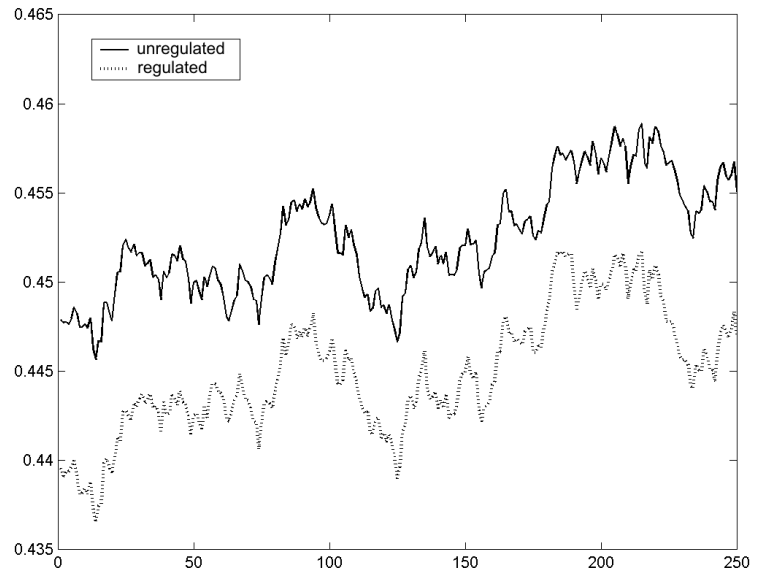

Figure A.3

Average correlation coefficient in 1000 simulations

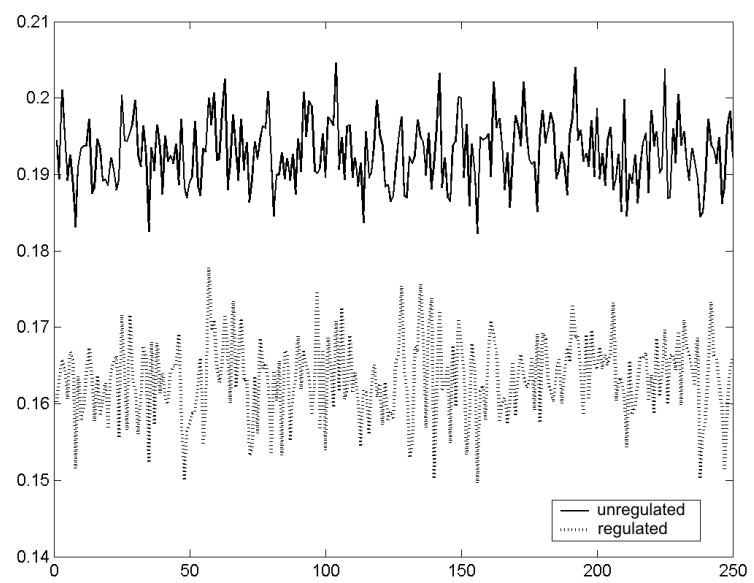

Figure A.4

Total bankruptcy probability 


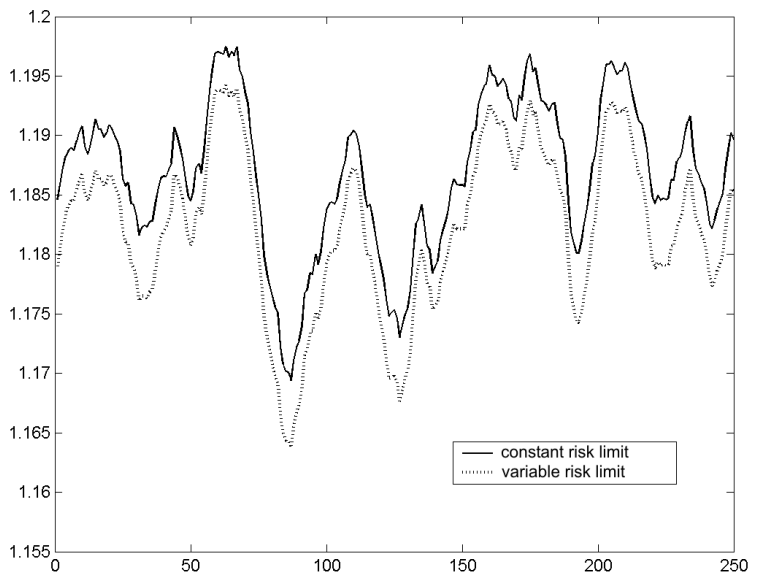

Figure A.5

Prices with constant and variable risk limits

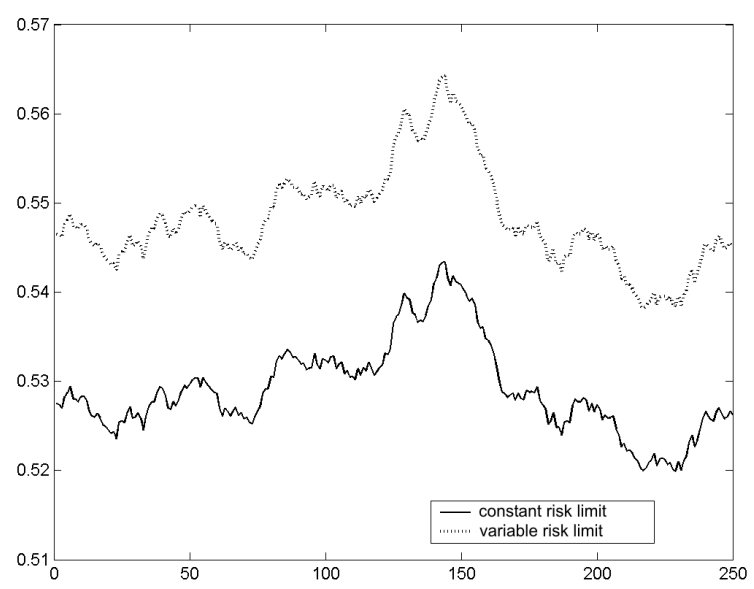

Figure A.6

Variances with constant and variable risk limits 


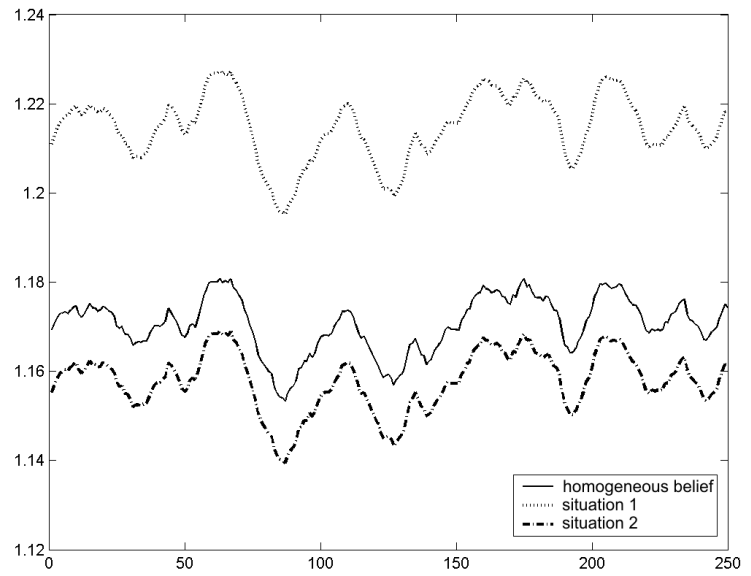

Figure A.7

Prices with homogeneous beliefs and in two extreme cases

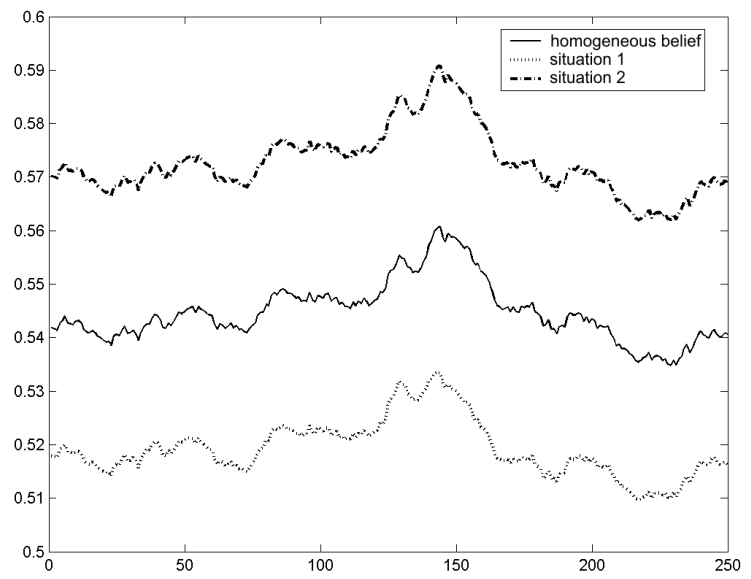

Figure A.8

Variances with homogeneous beliefs and in two extreme cases 


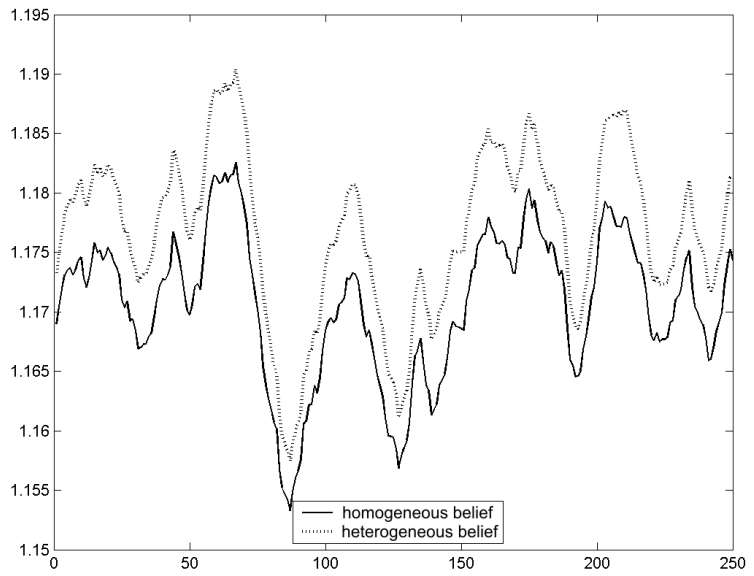

Figure A.9

Prices with homogeneous and heterogeneous beliefs

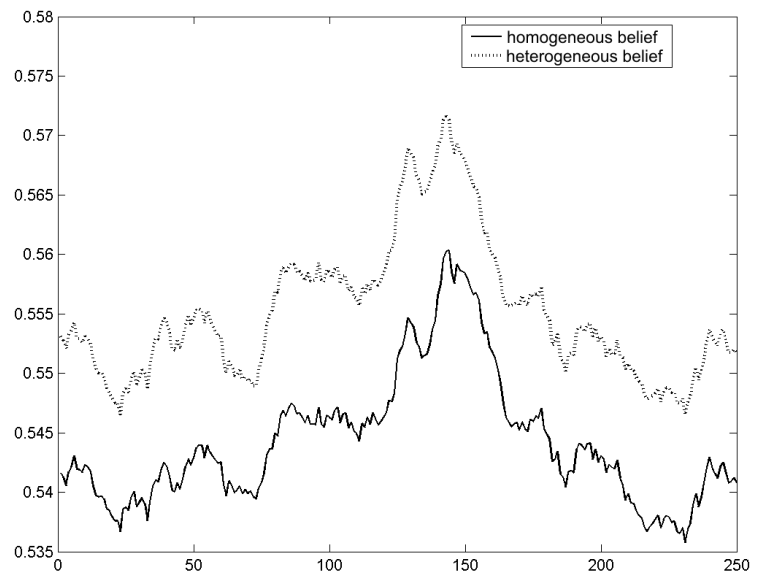

Figure A.10

Variances with homogeneous and heterogeneous beliefs 